\title{
A Research of Combining Curriculum with Practice of Food Nutriology Educational Reform
}

\author{
Yao Lv ${ }^{1,2}$, Qingsong Li ${ }^{1,3}$, Ruokun $\mathrm{Yi}^{1,3}$, and Xin Zhao ${ }^{1,3, *}$ \\ ${ }^{1}$ Chongqing Collaborative Innovation Center for Functional Food, Chongqing University of \\ Education, Chongqing 400067; \\ ${ }^{2}$ College of Tourism, Chongqing University of Education, Chongqing 400065; \\ ${ }^{3}$ College of Biological and Chemical Engineering, Chongqing University of Education, \\ Chongqing 400067
}

The corresponding author: Xin Zhao, zhaoxin@cque.edu.cn

\begin{abstract}
Food nutriology is an important professional basic course for cultivating application-oriented talents of food science and engineering, which is highly social, applicable and scientific. It will develop more subdisciplines with the development and intersection of subjects. As a branch of nutriology, food nutriology mainly studies: the relationship between food nutrition and human body growth and health. Owing to the rapid growth of theories and technology of food nutriology as well as new requirements and ideas for talent cultivation, the traditional teching modes of this course are unable to undertake the task of qualified talent cultivation of the subject. Therefore, based on current teaching conditions of food nutriology and for an increase of teaching quality, the reform should implemented by combining teaching contents and modes of the subject with practice, thus help students enhance their practice ability and stimulate their active study attitudes.
\end{abstract}

Keywords: Food and nutrition; Education; Curriculum; Practice

\section{食品营养学教学改革课程与实践相结合的探索}

\author{
吕瑶 ${ }^{1,2}$, 李青嵩 ${ }^{1,3}$, 易若琨 ${ }^{1,3}$, 赵欣 ${ }^{1,3, *}$
}

( 1. 重庆第二师范学院 重庆市功能性食品协同创新中心, 重庆 400067; 2. 重庆第二师范学院 旅游与服

务管理学院，重庆 400065；3．重庆第二师范学院 生物与化学工程学院，重庆 400067）

摘要: 食品营养学是食品科学与工程应用型人才培养的一门重要专业基础课程, 该课程具有较强的社会性、应用性和科 学性。随着学科的发展和交叉, 食品营养学还会出现更多的分支学科。作为营养学的分支, 食品营养学主要研究: 食物、营 养与人体生长发育以及健康的关系。随着食品营养学理论和技术的快速发展, 以及人才培养的新要求和新理念, 该棵程的传 统教学模式已经不能满足该专业人才的培养。所以, 为了提高教学质量, 在对食品营养学教学现状的基础上, 应该从食品营 养学的教学内容、模式和实践相结合的教学改革。从而提高学生们积极主动的学习态度和实践动手能力。

关键词: 食品营养学; 教育; 课程; 实践

中图分类号： G619.21 文献标志码: A

引言

食品营养学主要研究食物、营养与人体生长发育和健康的关系, 以及提高食品营养价值的措施。包括 各类食物的化学组成、营养特点; 每类食物中常见食物的特点; 食物加工、烹调和储藏对食物营养成分的 影响等。食品营养学是一门重要的医学边缘科学，与人类的健康息息相关，具有较强的社会性、应用性和 科学性。随着社会经济的发展, 生活水平的提高, 人们对食品的要求也越来越高。该课程的传统教学模式 已经不能满足高等学校培养专业人才的需求了。因此, 要进行食品营养学教学改革。为了提高教学质量和 
培养更多相关人才，需要对食品营养学的课程与实践相结合的教学改革。

\section{1 食品营养学的现状}

当今社会, 我国高等院校在食品营养学教学中, 虽然采用多媒体和课本相结合的方式授课。因为食品 营养学课程知识理论性较强, 大多数老师任然使用传统的灌输式教学模式, 很难吸引同学们的注意 [1]。 食品营养学的教学方式以传统的教学模式为主, 在整个教学过程中, 主要是以老师讲解来传授知识, 同学 们被动地接受知识。考试内容都是书上仅有的知识点, 同学们在考试前一周才开始死记硬背来临时记住知 识应付考试, 考试过后什么都不记得, 到头来什么知识也没有学到。这种教学模式既不能保证老师们的教 学质量, 也不能培养专业性人才。而且, 食品营养学的传统教学方式主要是以理论知识为主, 缺乏实际应 用。同学们所学的都是书本上的理论知识, 并没有真正掌握到食品营养学的应用技术和实践方法。很大程 度上限制了学生们的思考能力和实践动手能力 [2]。所以, 食品营养学的教学改革和提高同学们的实践能 力是非常有必要的。

\section{2 食品营养学课程与实践相结合的必要性}

\section{1 食品营养学课程落后于科学发展}

食品营养学课程现在仅有的教学内容相对比较陈旧, 教材中对很多营养素的功能介绍依然停留在很多 年前的水平上, 有的知识和基础理论与 “食品化学”、“生物化学” 等相关课程有很大部分存在重复。例 如: 营养物质的分类和结构上, 在相关课程上都有出现, 所以没有突出食品营养学本身的特点。而且, 对 食品营养学的研究领域及其成果非常少, 把食品营养学与其它学科相结合以及本学科的实际运用的研究更 是少之又少 [3]。教师的教学方法比较传统, 无法满足现代学生们学校的需求, 使学生们对食品营养学课 程学习态度和积极性受到冲击。因此, 为了适应科学的发展, 食品营养学课程与实践相结合的教学改革是 非常有必要的。

\section{2 缺乏食品营养学的实践应用}

现有的食品营养学教学模式仍然是以理论知识为主, 缺乏实践应用。随着食品营养学知识的不断传播, 我们日常生活中与营养相关的问题随处可见。在食品营养学的课程中很少出现理论知识与实践应用相结合 的内容, 以及用食品营养学的理论知识解决实际问题。使很多缺乏解决实际问题的能力和独立思考分析问 题的能力。而且, 老师们布置的作业就只是与课程知识相关的理论考题, 把食品营养学作为一门单独的课 程授课。所以, 导致大多数同学们认为作业只不过是练习练习, 巩固一下所学的理论知识, 很难让同学们 学到更多的实践运用, 很难让同学们受到更高层次和综合性的培养, 缺乏实战能力, 不利于专业性人才的 培养。所以要加强食品营养学教学改革 [4]。

\section{3 食品营养学理论知识难以培养专业人才}

随着经济的快速发展, 人们对食品的要求越来越高。但是, 现在的食品类相关专业 “食品营养学” 的 课程仍然使用预防医学专业的 “营养与食品卫生学” 教材, 这些相关教材在食品专业上缺乏科学体系和实 践操作, 教学内容与食品营养学理论知识存在差异, 使同学们对食品营养学的认知缺乏深入的了解, 对食 品营养学理论知识的作用和地位了解不够明显。目前的教学内容就是人体营养不良症状、人体内的营养过 程进行介绍, 缺乏对食品在加工处理、存储和销售等过程中发生的营养价值变化规律的介绍。而且, 如何 运用食品营养学理论知识来解决食品工艺技术与营养加工的问题少之又少, 导致很多同学难以把所学的食 
品营养学理论知识运用到所遇到的食品相关的问题上。所以, 目前的食品营养学理论知识很难培养出专业 人才, 需要进行教学改革 $[5]$ 。

\section{3 食品营养学教学改革的措施}

\section{1 扩大实训、实验基地建设}

在学校外面, 要加强与地区教育机构和食品单位的合作, 扩大和巩固标准的、稳定的食品营养学的实 训、实践基地建设, 形成学生们能够自己参与到实际生产过程中的实践原则。例如: 重庆第二师范学院生 物与化学工程系组织学生们到四川成都旺旺集团、重庆天友乳业有限公司、重庆沁园实业有限公司和重庆 德庄实业有限公司等地方食品企业进行实习。在实践中不断培养学生们的工作意识和操作能力。在学校内, 利用高校的优势, 联合食品单位与企业运行相吻合的高仿真实训、实践基地。从实践教学目标出发, 根据 相关行业的发展特点, 积极扩大生产的综合实训、实践基地, 建立起学生取样、检测、观察等实训、实践 制度，让每一位同学都亲身去体验一下车间生产的操作，把理论知识与实践紧密结合，培养出该行业有用 的专业性食品人才 $[6]$ 。

\section{2 改善考核制度}

现在的考核主要是以考试分数为主，考核制度以甄选与选拔为主要目的，而合理、科学的考核制度是 以增强学生们综合素质能力和促进学生们身心健康发展为主, 传统的考核制度存在很多不足, 所以在食品 营养学教学改革中必须加强改善考核制度。科学合理的考核制度应当淡化甄选性和选拔性, 更应该加强学 生们的职业道德和综合能力的培养, 不要再有以前的陈旧思想, 以考试的分数高低来决定学生们的能力, 更应该从学生们的教学任务完成情况与平时实际表现进行考核。同时还采用多种考核方式, 例如: 自我评 价、小组评价、教师评价等方式对学生们进行综合素质培养。让学生们树立正确的世界观、人生观、价值 观，使其在今后的学习中更加注重提高综合素质和培养综合能力，在学习中实现自我价值 [7]。

\section{3 加强实践教学方法改革}

综合实训、综合设计课程通过任务驱动的项目教学, 学生团队合作, 老师积极引导, 达到学生独立进 行实践操作，综合运用所学知识，或者自主设计实训、实践项目的目标。根据项目的要求进行新方案、新 工艺、新产品的设计与开发。在评价标准、内容、形式等方面体现出专业的特色。对实训、实践基地实行 开放制度, 随时可以举办各种专业技能竞赛活动、学科兴趣小组活动、创新性科技活动、社团活动, 从而 调动学生们的主动性和激发学生们的积极性, 培养学生们研究型学习、合作学习和自主学习的习惯。并且 全面推行集中实践制, 使校企强强结合, 校企联合研究实训、实践方案, 实施全程项目实训企业行业定岗 实习制和企业托管制, 构建高校与社会企业相互合作的大平台, 推动学生们综合能力的发展。通过课堂理 论学习与社会实践相结合, 才能吸引更多的学生, 让同学们觉得学习有动力、课堂有吸引力 [8]。

\section{4 更新教学内容}

教学内容是课程的核心, 教学内容是否先进和合理直接影响着学习的效果。一本科学合理的教材不仅 要有一定的体系, 而且还有比其它书籍更全面的理论知识。现在的食品营养学快速发展, 其理论知识不断 地增多, 应用范围更加广泛, 与生物技术、食品、农业等学科的融合日益紧密。更新教学内容应该与学科 前沿动态、社会热点问题和生产紧密联系, 不断研究领域的新知识和新成果。把食品营养学与其它相关学 科相结合, 从而使学生们认识到将生物技术应用到食品营养学研究中。让同学们认识多种植物化学物的功 
效, 将其应用于预防疾病和功能性食品的生产已经成为食品营养学的研究领域之一。想方设法让同学们理 解和掌握这些食品营养学领域的最新理论知识和研究成果, 有利于激发同学们的兴趣, 开阔同学们的视野。 老师讲课时, 要突出食品营养学的特点和讲授的重点, 而且要处理好教学重点与充实的内容的关系 $[9,10]$ 。

\section{4 结语}

在中国大部分的高校中还缺乏完善的食品营养学教学体系，改革现有的食品营养学教育方式建设完善 的食品营养学教学体系能加强食品科学专业的建设, 推动食品相关人才的培养。

\section{5 致谢}

本研究由重庆高校创新团队建设计划（CXTDX201601040）和重庆第二师范学院教学改革研究项目 （ZXJG03）基金支持。

The present research was supported by the Program for Innovation Team Building at Institutions of Higher Education in Chongqing (CXTDX201601040) and the Teaching Reform Research Project Transformation Research Project of Chongqing University of Education (ZXJG03).

\section{参考文献:}

[1] 马先红，刘洋，连丽丽. 食品营养学课程教学模式改革的综述 $[J]$. 科技资讯，2015，13(35)：201-202.

[2] 聂小华，孙培龙，何晋浙, 等. 基于建构主义的《食品营养学》课程教学改革与实践探索 [J]. 食品与机械, 2015(1)： $275-280$

[3] 鲁晓翔, 王志娟, 薛璐, 等. 优化教学内容提高教学质量一以 “食品营养学” 课程教学改革为例 [J]. 农产品加工, 2016 (6) : 69-71.

[4] Slater J, Hinds A. University student perceptions of Home Economics: food and nutrition education[J]. International Journal of Home Economics, 2014, 7(2): 68-80.

[5] Range1 CN, Nunn R, Dysarz F, et al. Teaching and learning about food and nutrition through science education in Brazilian schools: an intersection of knowledge[J]. Ciência and Saúde Coletiva, 2014, 19 (9): 3915-3924.

[6] 李青嵩，邓朝芳，李贵节，等. 多学科实验、实训课程改革探索一以重庆第二师范学院食品质量与安全(儿童营养 与健康)专业为例 $[J]$. 石家庄职业技术学报, 2015(4)：67-70.

[7］赵红梅. 《食品营养学》课程的教学改革与探索 [J]. 中华少年，2016(5)：233.

[8] Jounghee L, Soyeon J, Gyeongah K, et al. Development of a food safety and nutrition education program for adolescents by applying social cognitive theory[J]. Osong Public Health and Research Perspectives, 2016, $7(4): 248-260$.

[9] Kanda S, Chino K, Yoshimoto YS, et al. The educational effect of shokuiku (food and nutrition education) taught in English as a form international exchange in Japanese elementary schools [J]. Japanese Journal of Health and Human Ecology, 2012, 78: 91-102.

[10] Webb M, Morancie A. Food safety knowledge of foodservice workers at a university campus by education level, experience, and food safety training[J]. Food Control, 2015, 50: 259-264.

\section{References:}

[1] Ma Xianhong, Yang Liu, Lian Lili. Summary of Teaching Mode Reform of Food Nutrition Course [J].Science and Technology Information, 2015, 13(35):201-202.

[2] Nie Xiaohua, Sun Peilong, He Jinzhe, and so on. Course Teaching Reform and Practice exploration of Food Nutrition Based on Constructivism [J].Food and Machinery, 
2015(1):275-280.

[3] Lu Xiaoxiang, Wang Zhijuan, Xue Lu, et al. Optimization of teaching Contents to Improve Teaching Quality-"Food Nutrition" Course Teaching Reform as An Example [J].Agricultural Products Processing, 2016(6):69-71.

[4] Slater J, Hinds A. University student perceptions of Home Economics: food and nutrition education [J]. International Journal of Home Economics, 2014, 7(2): 68-80.

[5] Rangel CN, Nunn R, Dysarz F, et al. Teaching and learning about food and nutrition through science education in Brazilian schools: an intersection of knowledge [J]. Ciência and Saúde Coletiva, 2014, 19(9): 3915-3924.

[6] Li Qingsong, Deng Chaofang, Li Guijie, and so on. Multidisciplinary Experiment and Practical Training Curriculum Reform-Food Quality and Safety (Child Nutrition and Health) of Chongqing Second Teachers College as An Example [J].Journal of Shijiazhuang Vocational Technology Institute, 2015(4):67-70.

[7] Zhao Hongmei. Teaching Reform and Exploration on Food Nutrition Course [J].China Juveniles, 2016(5):233.

[8] Jounghee L, Soyeon J, Gyeongah K, et al. Development of a food safety and nutrition education program for adolescents by applying social cognitive theory[J]. Osong Public Health and Research Perspectives, 2016, 7(4): 248-260.

[9] Kanda S, Chino K, Yoshimoto YS, et al. The educational effect of shokuiku (food and nutrition education) taught in English as a form international exchange in Japanese elementary schools [J]. Japanese Journal of Health and Human Ecology, 2012, 78: 91-102.

[10] Webb M, Morancie A. Food safety knowledge of foodservice workers at a university campus by education level, experience, and food safety training [J]. Food Control, 2015, 50: 259-264. 